\title{
FEMTOSECOND LINAC-LASER BASED TIME-RESOLVED X-RAY DIFFRACTOMETRY FOR VISUALIZATION OF ATOMIC MOTIONS
}

\author{
K. Kinoshita, H. Harano, S. Okita, T. Ueda, M. Uesaka, T. Watanabe and K. Yoshii \\ Nuclear Engineering Research Laboratory, University of Tokyo, \\ Tokai-mura, Naka-gun, Ibaraki-ken, Japan
}

\begin{abstract}
We carried out the time-resolved X-ray diffraction experiment where 100fs 3TW laser and 10ps X-rays are used as pump-and probe-pulses, respectively. We used the synchronized femtosecond electron linac and laser system. Using 10ps $\mathrm{Cu} \mathrm{K} \alpha 1,2 \mathrm{X}$-ray pulses via collision between 10ps electron beam and a $\mathrm{Cu}$ wire, we could obtain diffraction images from several monocrystal semiconductors $(\mathrm{Si}, \mathrm{GaAs}, \mathrm{Ge})$, ion-crystals $(\mathrm{NaCl}, \mathrm{KCl})$ and alkalihalides $(\mathrm{CaF} 2, \mathrm{BaF} 2)$. However, the change of the X-ray diffraction image could not be observed because of surface damage due to repeated laser irradiation. In order to get more X-ray photons and perform single-shot pump-and-probe analysis, we are going to proceed to laser plasma X-ray based analysis.
\end{abstract}

\section{INTRODUCTION}

Synchronized femtosecond electron and laser beams can be generated and measured at Nuclear Engineering Research Laboratory, University of Tokyo[1,2,3]. Those primary femtosecond beams are converted to far-infrared coherent radiation and X-ray pulses via transition radiation and collision with metal targets, respectively. Thus we can perform ultrashort quantum beam based pump-and-probe analysis to investigate ultrafast beammatter interactions. Here atomic motions in nonequilibrium thermal expansion, ablation, phase transition, soft-mode phonon are expected to be observed as snapshots of X-ray diffraction images. We proposed the new time-resolved X-ray diffractometry where ultrashort X-rays via electron-metal collision is used as a probe pulse[4]. X-ray diffraction images from several monocrystals using 10ps $\mathrm{Cu} \mathrm{K} \alpha 1,2 \mathrm{X}$-rays are presented and future subjects to be overcome are mentioned.

\section{LINAC BASED EXPERIMENT}

Experimental configuration is depicted in Fig.1. $35 \mathrm{MeV}$, 10ps(FWHM), 1nC electron single bunch was irradiated with the $100 \mu \mathrm{m}^{\mathrm{t}} \mathrm{Cu}$ wire to generate $\mathrm{Cu} \mathrm{K} \alpha 1,2 \mathrm{X}$ rays $(8.048,8.028 \mathrm{keV})$. Generation of $10 \mathrm{ps} \mathrm{Cu} \mathrm{K} \alpha 1,2 \mathrm{X}$ - rays was confirmed by the numerical analysis using EGS4 code[4]. The 100fs 3TW Ti:Sapphire laser[5] was used as a pump pulse to induce nonequilibrium thermal expansion. Here the linac-laser synchronization system[1] was used to control the delay time of the probe-X-rays from the pump-laser.

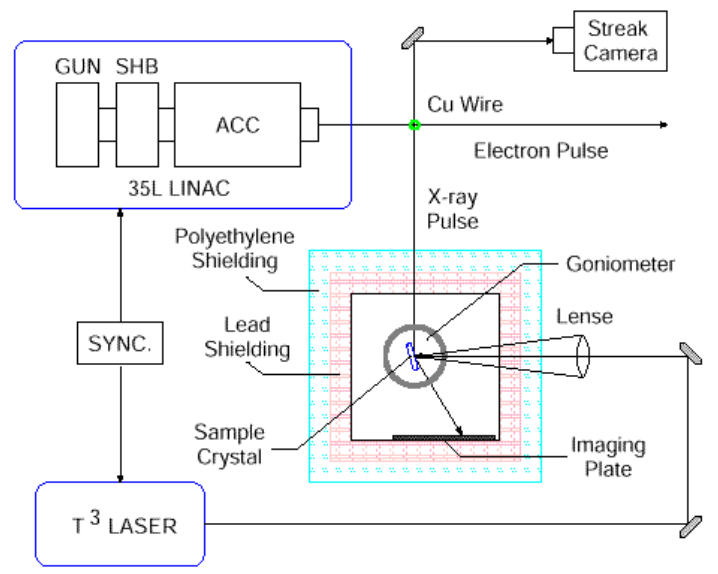

Figure 1: Configuration of the linac-based experiment.

We used monocrystal semiconductors of $\mathrm{Si}(111)$, GaAs (111), Ge (111), ion crystals of $\mathrm{NaCl}$ (200) $\mathrm{KCl}(200)$ and monocrystal alkalihalides $\mathrm{CaF}_{2}(220)$, $\mathrm{BaF}_{2}(111)$. X-ray diffraction image is drawn on an X-ray imaging plate set in the noise-radiation shielding box. Laser energy per shot is $68 \mathrm{~mJ}$. The number of photons of the X-ray per shot is estimated to be about $10^{6}$. Due to the limited solid angle at a specimen, only about $1 / 100$ of them reach the specimen. Thus, we need $10^{4}-10^{5}$ times repetition for the pump-and-probe shot. $\mathrm{Cu} \mathrm{K \alpha 1,2} \mathrm{X}$-ray diffraction images from all specimens were successfully obtained. The pump-and-probe analysis was carried out for a GaAs specimen. However, its surface suffered laser-irradiation damage before the X-ray diffraction image for deformed lattice was obtained. Photograph of the damaged GaAs specimen and the $\mathrm{Cu} \mathrm{K} \alpha 1,2$ X-ray diffraction images influenced by the damage are shown 
in Fig.2. The damage may attribute physical ablation or photo-chemical reaction with air.

Let us clarify the characteristics of the electron linac based X-ray diffractometry. Based on the numerical analysis by EGS4, subpicosecond $\mathrm{Cu} \mathrm{K} \alpha 1,2$ X-rays can be surely produced. However, due to lack of photon numbers, we need many times pump-and-probe shots to get the X-ray diffraction image. In this case, the phenomena to be observed have to be nondestructive. If the phenomena is destructive, we should choose the TW laser plasma based one.
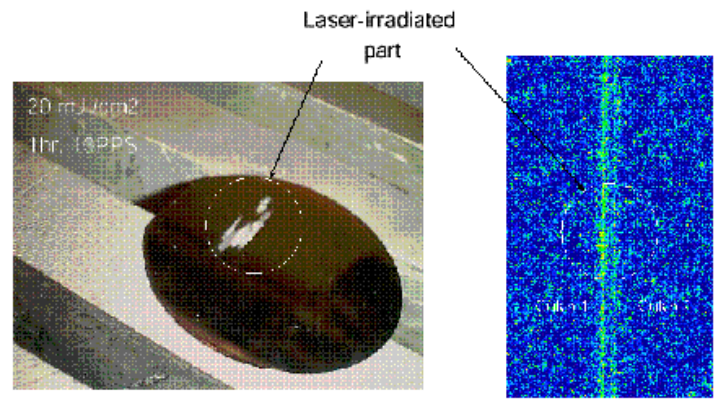

GaAs wafer with damage due to laser irradiation

Figure 2: GaAs specimen with damage and K $\alpha 1,2$ $\mathrm{X}$-ray diffraction image.

\section{TW FS LASER BASED EXPERIMENT}

When a TW fs laser is irradiated at a $\mathrm{Cu}$ plate, the ablation occurs and the plasma is generated. Then hot electrons over $10 \mathrm{keV}$ are also produced there. These electrons can induce $\mathrm{Cu} K \alpha 1,2$ X-rays. The main advantage of this process is a large number of X-rays photons as about $10^{11}$ per shot, while the drawback is its long pulse width as a few ps[7]. In this case, it is expected that one laser-and-X-ray irradiation is enough to get a diffraction image. We carried out $\mathrm{Cu} \mathrm{K} \alpha 1,2 \mathrm{X}$ rays generation via this process using the $2 \mathrm{TW} 100 \mathrm{fs}$ laser light. The generation was confirmed by depicting the characters of "LPX" on the image plate as shown in Fig.3. We are going to use the laser induced plasma Xrays in the next step.

\section{NUMERICAL ANALYSIS}

We are developing a computer code to calculate the change of $\mathrm{Cu} \mathrm{K \alpha 1,2} \mathrm{X}$-rays diffraction image due to atomic motions. We have already calculated the images of deformed lattices under several assumptions. Then, we are going to calculate the change of the image due to realistic nonequilibrium thermal expansion, phasetransition and soft-mode phonon. By using this code, we plan to perform an inverse analysis to evaluate deformed lattice from measured X-ray diffraction images in the pump-and-probe analysis. The final goal is the animation of the atomic motions via computer graphics.

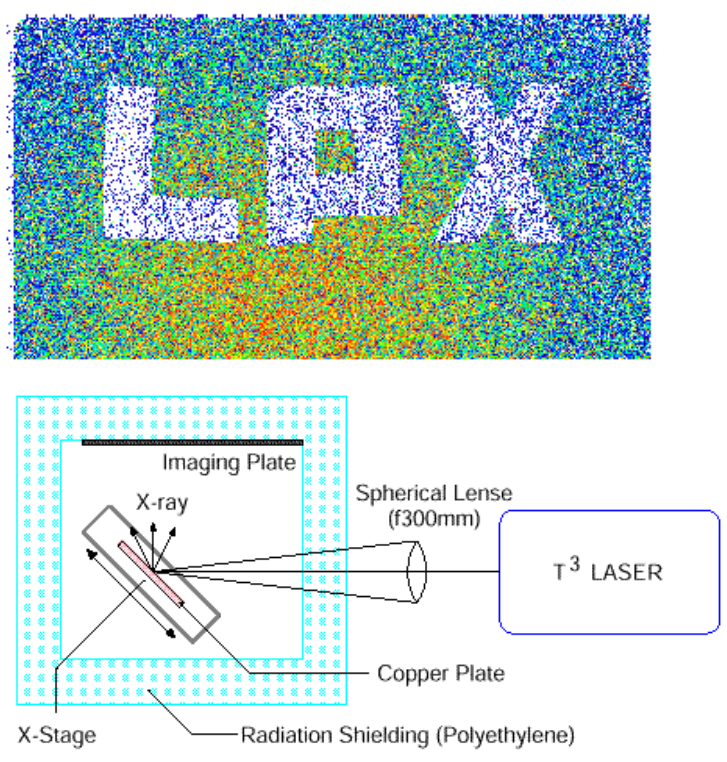

Figure 3: Configuration of the TW FS laser based experiment and result.

\section{FEMTOSECOND ULTRAFAST QUANTUM PHENOMENA RESEARCH FACULTY}

The titled facility is going to be installed in the laboratory. Here the upgraded femtosecond S-band twin linacs with 100fs stable Kerr-lens-model-locked Ti:Sapphire laser, 12TW50fs laser, X-ray diffraction analysis devices, the X-ray electron spectroscopy device and the Fourier transform infra-red spectroscopy device are introduced as shown in Fig.4.

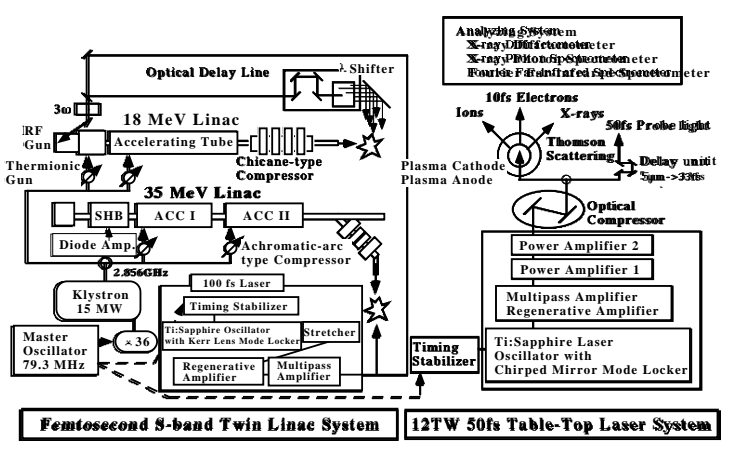

Fig.4 Femtosecond ultrafast quantum phenomena research facility. 
Hundreds fs time-resolved pulseradiolysis for radiation chemistry is available using the first, while several basic researches of tens fs beam (electron, ion, X-rays) generation are performed via plasma cathode, plasma anode and relativistic nonlinear Thomson scattering using the second. After we have succeeded in the generation experimentally, we can proceed to a variety of tens fs time-resolved pump-and-probe analyses.

\section{CONCLUSION}

10ps $\mathrm{Cu} \mathrm{K} \alpha 1,2 \mathrm{X}$-rays was generated via 10ps elections$\mathrm{Cu}$ wire collision using the electron linac. $\mathrm{Cu} \mathrm{K} \alpha 1,2 \mathrm{X}$ rays diffraction images were obtained for several monocrystals. Further, we produced picoseconds laser plasma $\mathrm{Cu} \mathrm{K \alpha 1,2} \mathrm{X}$-rays via $2 \mathrm{TW} 100 \mathrm{fs}$ laser-Cu plate irradiation. In order to get $\mathrm{X}$-ray diffraction images from deformed lattices in laser-induced nonequilibrium thermal expansion by single pump-and-probe shot, we plan to use the new 12TW 50fs laser in the next step.

\section{REFERENCE}

[1] M. Uesaka et al., J. Nucl. Mater., 248, 380(1997).

[2] M. Uesaka et al., Nucl. Instrum. Meth., A, 406, 371(1998).

[3] T. Watanabe et al., Proc. of EPAC98, 1632(1998).

[4] H. Harano et al., ibid, 2452(1998).

[5] K. Nakajima et al., AIP Coference Proceedings 398, 83(1997).

[6] C. Barty et al., http://www-wilson.ucsd.edu.

[7] K. Kondo et al., Appl. Phys. Lett., 73(17), 1(1998). 